\title{
Scattering and bound states for the Hulthén potential in a cosmic string background
}

\author{
Mansoureh Hosseinpour ${ }^{1, a}$, Fabiano M. Andrade ${ }^{2, b}$, Edilberto O. Silva ${ }^{3, c}$, Hassan Hassanabadi ${ }^{1, d}$ \\ ${ }_{1}^{1}$ Physics Department, Shahrood University of Technology, P. O. Box: 3619995161-316, Shahrood, Iran \\ ${ }^{2}$ Departamento de Matemática e Estatística, Universidade Estadual de Ponta Grossa, 84030-900 Ponta Grossa, PR, Brazil \\ ${ }^{3}$ Departamento de Física, Universidade Federal do Maranhão, 65085-580 São Luís, MA, Brazil
}

Received: 20 January 2017 / Accepted: 15 April 2017 / Published online: 28 April 2017

(C) The Author(s) 2017. This article is an open access publication

\begin{abstract}
In this work we study the Dirac equation with vector and scalar potentials in the spacetime generated by a cosmic string. Using an approximation for the centrifugal term, a solution for the radial differential equation is obtained. We consider the scattering states under the Hulthén potential and obtain the phase shifts. From the poles of the scattering $S$-matrix the states energies are determined as well.
\end{abstract}

\section{Introduction}

To study the relativistic quantum dynamics of particles with spin under the influence of electromagnetic fields in curved spacetime we must consider the modified covariant form of the Dirac equation $(\hbar=c=1)[1,2]$

$$
\left\{i \gamma^{\mu}(x)\left[\partial_{\mu}+\Gamma_{\mu}(x)+i e A_{\mu}(x)\right]-M\right\} \psi(x)=0
$$

where $A_{\mu}$ denotes the vector potential associated with the electromagnetic field, $\Gamma_{\mu}(x)$ is the spinor affine connection and $\gamma^{\mu}(x)$ are the Dirac matrices in the curved spacetime. The $\gamma^{\mu}(x)$ matrices are constructed from the standard Dirac matrices in Minkowski spacetime, which are written in terms of local coordinates, and we write them in terms of global coordinates using the inverse vierbeins $e_{a}^{\mu}(x)$ through the relation

$\gamma^{\mu}(x)=e_{a}^{\mu}(x) \gamma^{a}, \quad(\mu, a=0,1,2,3)$,

\footnotetext{
a e-mail: hosseinpour.mansoureh@gmail.com

be-mail: fmandrade@uepg.br

c e-mail: edilbertoo@gmail.com

d e-mail: h.hasanabadi@shahroodut.ac.ir
}

with $\gamma^{a}=\left(\gamma^{0}, \gamma^{i}\right)$ being the standard gamma matrices. The $\gamma^{\mu}(x)$ matrices also define the covariant Clifford algebra,

$\left\{\gamma^{\mu}(x), \gamma^{\nu}(x)\right\}=2 g^{\mu \nu}(x)$.

If we want the particle to interact with scalar potentials it is more convenient to write the Dirac equation (1) in the following form [3]:

$$
\left\{\alpha^{i}\left[p_{i}-i \Gamma_{i}-e A_{i}\right]+e A_{0}-i \Gamma_{0}+\beta M\right\} \psi(x)=E \psi(x),
$$

for $i=1,2,3$, and then introduce the vector and scalar couplings,

$$
\begin{aligned}
E & \rightarrow E-V(r), \\
M & \rightarrow M+S(r),
\end{aligned}
$$

respectively.

It is important to note that these couplings differ in the manner how they are inserted into the Dirac equation. In Ref. [4] it was shown how the vector coupling in (5) acts on electron and positron states. As a result, the potential couples to the charge and a great number of physical phenomena can be studied through the Dirac equation (1). For instance, it is used to study the relativistic quantum motion of charged spin- 0 and spin- $1 / 2$ particles in the presence of a uniform magnetic field and scalar potentials [5], to study the influence of topological defects on the spin current as well as the spin Hall effect [6] and to investigate the role of the cosmic string on spin current and Hall electric field [7]. On the other hand, contrary to the coupling (5), the scalar coupling (6) acts equally on particles and antiparticles. Namely, we can add it directly to the mass term of the Dirac equation. The most common interpretation known in the literature for this coupling is that of a position-dependent effective mass [8]. 
This coupling has been used, for example, to study the problem of a relativistic particle with position-dependent mass in the presence of a Coulomb and a scalar potential in the background spacetime generated by a cosmic string [9].

These couplings are also used to study various physical models by including interactions and then to investigate their possible physical implications on system dynamics. For example, the Aharonov-Bohm effect [10,11], the Dirac particle in a Morse potential [12,13], the Dirac equation for an attractive Coulomb potential in supersymmetric quantum mechanics [14], the Dirac equation with scalar and vector potentials under the exact spin and pseudospin symmetries limits [15-18] and particles interacting with the Hulthén [19] and Rosen-Morse [20] potentials.

Among the most important problems that we can study using the couplings (5) and (6), we refer to the scattering problems. The scattering problems of quantum systems, i.e., the prediction of reaction probabilities when two objects collide, provide us with a reliable understanding of the physical system under investigation and they belong to the most effective ways to study the structure of matter. Great parts of our current theories are built on the basis of scattering experiments. The best-known example of this claim is the atomic nucleus. Scattering problems are among the most technically demanding problems in quantum physics. The underlying difficulty lies in the unbounded nature of the wave function in these problems. The process of scattering of particles by potentials changes their wave function by introducing phase shifts. The study of these phase shifts allow us to predict experimental observations from the fundamental interactions postulated by the theory. These studies have already been done in nonrelativistic and relativistic quantum mechanics [4,21-26]. Nevertheless, the dominant part of these scattering studies is limited to flat spacetime and the cases regarding the curved spacetime are actually less frequent. On the other hand, the dynamics of quantum mechanics on curved spaces in the presence of topological defects has attracted much attention in recent years [27-35].

In the present work, we are going to consider the scattering problem of the Dirac equation produced by the Huthén potential in a cosmic string background. As will be showed, due to the presence of the centrifugal term in the radial differential equation, it cannot be solved in an exact manner. An approximation is used in order to obtain an approximated solution for the problem. The cosmic string is a linear defect that changes the topology of the medium. This field has been an appealing research field in the past years as not much is known in comparison with the ordinary Dirac equation in flat space [36-41]. From the field theory point of view, the cosmic string can be viewed as a consequence of symmetry breaking phase transition in the early Universe [42]. Until now some problems have been investigated in curved spacetime including the one-electron atom problem [43-45]. The dynamics of non-relativistic particles in curved spacetime is considered in $[29,30,46-50]$ as well.

This paper is organized as follows. In Sect. 2, we study the covariant Dirac equation in the spacetimes generated by a cosmic string in the presence of vector and scalar potentials of electromagnetic field. We then find special cases of the equation for equal and opposite scalar and vector potentials. In Sect. 3, we consider the Dirac equation with the Hulthén potential in the context of spin and pseudo-spin symmetries and obtain the scattering solutions as well as the phase shifts. In Sect. 4, we derive the scattering $S$-matrix and from its poles we determine the bound state energies. Finally, in Sect. 5, we present our conclusions.

\section{Dirac equation in cosmic string background}

The line element corresponding to the cosmic string spacetime $[51,52]$ is given, in spherical coordinates, by $[53,54]$

$\mathrm{d} s^{2}=-\mathrm{d} t^{2}+r^{2} \mathrm{~d} \theta^{2}+\alpha^{2} r^{2} \sin ^{2} \theta \mathrm{d} \varphi^{2}$,

where $t \in(-\infty, \infty), r \in[0, \infty), \theta \in[0, \pi / 2]$ and $\phi \in$ $[0,2 \pi]$. The $\alpha$ parameter in Eq. 7 is related to the linear mass $\bar{\mu}$ of the string by $\alpha=1-4 \bar{\mu}$ and it is defined in the range $(0,1]$.

Now, in order to write the Dirac equation (4) in the cosmic string spacetime, we must rewrite the Dirac matrices in terms of global coordinates. Additionally, we have to calculate the affine spinorial connection $\left(\Gamma_{0}, \Gamma_{i}\right)$. The details of this calculation can be found in $[3,24]$. By using the wave function decomposition in the form

$\psi(x)=e^{-i E t} \chi(r, \theta, \varphi)$,

the Dirac equation in (4) can be written as

$$
\begin{aligned}
& \left\{i \alpha^{r} \partial_{r}+\frac{i \alpha^{\theta}}{r} \partial_{\theta}+\frac{i}{\alpha r \sin \theta} \alpha^{\varphi} \partial_{\varphi}\right. \\
& \quad+\frac{i}{2 r}\left(1-\frac{1}{\alpha}\right)\left(\alpha^{r}+\cot \theta \alpha^{\theta}\right) \\
& \left.\quad-\gamma^{0}[M+S(r)]+E-V(r)\right\} \chi(r, \theta, \varphi)=0,
\end{aligned}
$$

where we have included a scalar $M \rightarrow M+S(r)$ and a vector $E \rightarrow E-V(r)$ coupling. Now, assuming that the solutions of Eq. (9) are of the form [45]

$$
\chi(r, \theta, \varphi)=r^{-(\alpha-1) / 2 \alpha}(\sin \theta)^{-(\alpha-1) / 2 \alpha} F(r) \Theta(\theta) \Phi(\varphi),
$$


we find the radial equation

$$
\begin{aligned}
& \left\{\tilde{\alpha}^{r} p_{r}+\frac{i}{r} \tilde{\alpha}^{r} \sigma^{z} \boldsymbol{\kappa}_{(\alpha)}+V(r)+[M+S(r)] \sigma^{z}\right\} F_{n, \kappa_{(\alpha)}}(r) \\
& =E F_{n, \kappa_{(\alpha)}}(r)
\end{aligned}
$$

with [45]

$\tilde{\alpha}^{r}=\left(\begin{array}{cc}0 & -i \\ i & 0\end{array}\right), \quad \sigma^{z}=\left(\begin{array}{cc}1 & 0 \\ 0 & -1\end{array}\right)$.

In Eq. (11), $\boldsymbol{\kappa}_{(\alpha)}$ represents the generalized spin-orbit operator in the spacetime of a cosmic string whose eigenvalues are given by

$\kappa_{(\alpha)}= \pm\left[j_{(\alpha)}+\frac{1}{2}\right]= \pm\left[j+m\left(\frac{1}{\alpha}-1\right)+\frac{1}{2}\right]$

with $j_{(\alpha)}$ representing the eigenvalues of the generalized total angular momentum operator. The operator $\boldsymbol{\kappa}_{(\alpha)}$ is given by

$\sigma^{z} \boldsymbol{\kappa}_{(\alpha)}=\tilde{\boldsymbol{\alpha}} \cdot \mathbf{L}_{(\alpha)}+1$,

where $\mathbf{L}_{(\alpha)}$ is the generalized angular momentum operator in the spacetime of the cosmic string,

$L_{(\alpha)}^{2} Y_{\ell_{(\alpha)}}^{m_{(\alpha)}}(\theta, \varphi)=\ell_{(\alpha)}\left(\ell_{(\alpha)}+1\right) Y_{\ell_{(\alpha)}}^{m_{(\alpha)}}(\theta, \varphi)$,

with $Y_{\ell_{(\alpha)}}^{m_{(\alpha)}}(\theta, \varphi)$ being the generalized spherical harmonics and $m_{(\alpha)}$ and $\ell_{(\alpha)}$ not necessarily being integers. In particular $m_{(\alpha)}=m / \alpha$, where $m=0, \pm 1, \pm 2, \ldots, \alpha \in(0,1]$ and $\ell_{(\alpha)}=n+m_{(\alpha)}=\ell+m(1 / \alpha-1), \ell=0,1,2, \ldots, n-1$. Here $\ell$ and $m$ are, respectively, the orbital angular momentum quantum number and the magnetic quantum number in the flat space (i.e., for $\alpha=1$ ), and $n$ is the principal quantum number.

By choosing the radial wave function as [45]

$F(r)=\frac{1}{r}\left(\begin{array}{c}-i f(r) \\ g(r)\end{array}\right)$,

we obtain the coupled equations

$$
\begin{aligned}
& -i[E-M-(S+V)] f(r)+\frac{\mathrm{d} g(r)}{\mathrm{d} r}+\frac{\kappa_{(\alpha)}}{r} g(r)=0, \\
& -i[E+M+S-V] g(r)+\frac{\mathrm{d} f(r)}{\mathrm{d} r}+\frac{\kappa_{(\alpha)}}{r} f(r)=0 .
\end{aligned}
$$

Let us now consider the special case of $S(r)=V(r)$ (exact spin symmetry limit) and $S(r)=-V(r)$ (exact pseudo-spin symmetry limit). After elimination of one component in favor of the other, for $S(r)=V(r)$, we have

$$
\begin{aligned}
-\frac{\mathrm{d}^{2} f(r)}{\mathrm{d} r^{2}}+ & {\left[\frac{\kappa_{(\alpha)}\left(\kappa_{(\alpha)}-1\right)}{r^{2}}\right.} \\
& -(E-M-2 V(r))(E+M)] f(r)=0 .
\end{aligned}
$$

Additionally, for the case $S(r)=-V(r)$, we obtain

$$
\begin{aligned}
-\frac{\mathrm{d}^{2} g(r)}{\mathrm{d} r^{2}}+ & {\left[\frac{\kappa_{(\alpha)}\left(\kappa_{(\alpha)}+1\right)}{r^{2}}\right.} \\
& -(E+M-2 V(r))(E-M)] g(r)=0 .
\end{aligned}
$$

Thus comparing Eqs. (19) and (20), we can see that the solution for the case $S(r)=-V(r)$ can be obtained from the solution for the case $S(r)=V(r)$ with the replacements $\kappa_{(\alpha)}-1 \rightarrow \kappa_{(\alpha)}+1$ and $M \rightarrow-M$. Therefore we shall only deal with the latter because the results for the former can be obtained in a straightforward manner by using the above replacements.

\section{Scattering analysis}

In this work, we are interested in considering the Hulthén potential, which has remarkable applicabilities because of its short-range nature. It should be noted that this potential is a special case of the Eckert potential [55]. Therefore, we are going to investigate scattering state solutions of the Dirac equation in the presence of the Hulthén potential,

$V(r)=-\xi \frac{\omega}{e^{\omega r}-1}$,

where $\omega$ is the screening parameter and $\xi$ is a positive constant. When $V(r)$ is used to describe atomic phenomena, $\xi$ is interpreted as $Z e^{2}$, with $Z$ the atomic number. In this step, we want to evaluate phase shifts and normalization factor for the pseudo-spin symmetry limit (i.e., $S(r)=V(r)$ ). Thus inserting Eq. (21) into (19), we obtain

$$
\begin{aligned}
& {\left[-\frac{\mathrm{d}^{2}}{\mathrm{~d} r^{2}}+\frac{\kappa_{(\alpha)}\left(\kappa_{(\alpha)}-1\right)}{r^{2}}-2(E+M) \xi \frac{\omega e^{-\omega r}}{1-e^{-\omega r}}\right] f(r)} \\
& \quad=k^{2} f(r),
\end{aligned}
$$

where $k^{2}=E^{2}-M^{2}$. It is worthwhile to note here that, for small values of $\omega$, the Hulthén potential behaves like the Coulomb potential, consequently Eq. (22) turns into the Bessel equation [56-59]. In contrast with the Coulomb potential, unfortunately, the Dirac equation for the Hulthén potential cannot be solved analytically due to the presence of the 
centrifugal term. In this manner, it is necessary to use some an approximation. Considering small values of $\omega$, a common approximation for the centrifugal term is

$\frac{1}{r^{2}} \approx \frac{\omega^{2} e^{-\omega r}}{\left(1-e^{-\omega r}\right)^{2}}$

The above approximation, as we shall see, leads us to a solvable differential equation $[55,60]$. Therefore, by using the approximation in Eq. (23), followed by the change of variable $y=1-e^{-\omega r}$, we can write Eq. (22) in the form

$$
\begin{aligned}
& {\left[\frac{\mathrm{d}^{2}}{\mathrm{~d} y^{2}}-\frac{1}{1-y} \frac{\mathrm{d}}{\mathrm{d} y}-\frac{\kappa_{(\alpha)}\left(\kappa_{(\alpha)}-1\right)}{y^{2}(1-y)}+\frac{\eta^{2}}{y(1-y)}\right.} \\
& \left.\quad+\frac{\varepsilon^{2}}{(1-y)^{2}}\right] f(y)=0,
\end{aligned}
$$

in which $\eta^{2}=2(E+M) \xi / \omega$ and $\varepsilon^{2}=k^{2} / \omega^{2}$. Equation (24) can be turned into a well-known differential equation if we choose

$$
f(y)=y^{\gamma}(1-y)^{v} h(y)
$$

as the solutions, where $v$ and $\gamma$ are arbitrary constants to be determined. Therefore, substituting Eq. (25) into (24) we obtain

$$
\begin{aligned}
& {\left[\frac{\mathrm{d}^{2}}{\mathrm{~d} y^{2}}+\frac{2 \gamma-(1+2 v+2 \gamma) y}{y(1-y)} \frac{\mathrm{d}}{\mathrm{d} y}+\frac{v^{2}+\varepsilon^{2}}{(1-y)^{2}}\right.} \\
& \left.+\frac{\eta^{2}-2 \gamma v-\gamma^{2}}{y(1-y)}+\frac{\gamma(\gamma-1)-\kappa_{(\alpha)}\left(\kappa_{(\alpha)}-1\right)}{y(1-y)^{2}}\right] h(y)=0 .
\end{aligned}
$$

We determine the parameters $v$ and $\gamma$ by imposing that the coefficients of the terms $1 /(1-y)^{2}$ and $1 /\left[y(1-y)^{2}\right]$ vanish identically. In this manner, we have

$v= \pm \frac{i k}{\omega}, \quad \gamma=\kappa_{(\alpha)}$ or $\gamma=1-\kappa_{(\alpha)}$.

This set of parameters leads us to the same solution for Eq. (26) and we are free to choose one set. Thus choosing $v=$ $i k / \omega$ and $\gamma=\kappa_{(\alpha)}$ this leads to

$$
\left\{y(1-y) \frac{\mathrm{d}^{2}}{\mathrm{~d} y^{2}}+\left[\eta_{3}-\left(1+\eta_{1}+\eta_{2} y\right] \frac{\mathrm{d}}{\mathrm{d} y}-\eta_{1} \eta_{2}\right\} h(y)=0\right.
$$

where

$$
\begin{aligned}
& \eta_{1}=\gamma+v+\sqrt{\eta^{2}+v^{2}}=\kappa_{(\alpha)}+\frac{i k}{\omega}+\sqrt{\frac{2(E+M) \xi}{\omega}-\frac{k^{2}}{\omega^{2}}}, \\
& \eta_{2}=\gamma+v-\sqrt{\eta^{2}+v^{2}}=\kappa_{(\alpha)}+\frac{i k}{\omega}-\sqrt{\frac{2(E+M) \xi}{\omega}-\frac{k^{2}}{\omega^{2}}}, \\
& \eta_{3}=2 \gamma=2 \kappa_{(\alpha)} .
\end{aligned}
$$

Equation (28), has the form of a hypergeometric differential equation [44],

$h(y)={ }_{2} F_{1}\left(\eta_{1}, \eta_{2}, \eta_{3} ; y\right)$.

Therefore, the radial wave functions can be written as

$$
f(r)=N\left(1-e^{-\omega r}\right)^{\ell} e_{2}^{i k r} F_{1}\left(\eta_{1}, \eta_{2}, \eta_{3} ; 1-e^{-\omega r}\right) .
$$

Now, in order to obtain the scattering phase shift and the normalization factor we write the asymptotic form of the above wave function. For this purpose we use the properties of the hypergeometric functions [61] and the asymptotic behavior of (31) to $r \rightarrow \infty$ [24]

$$
\begin{aligned}
f(r) \sim & 2 N\left[\Gamma\left(\eta_{3}\right)\right]^{2}\left|\frac{\Gamma\left(\eta_{3}-\eta_{1}-\eta_{2}\right)}{\Gamma\left(\eta_{3}-\eta_{1}\right) \Gamma\left(\eta_{3}-\eta_{2}\right)}\right| \\
& \times \sin \left(k r+\frac{\pi}{2}+\delta\right)
\end{aligned}
$$

where $N$ is a normalization constant. Recalling the boundary condition for $r \rightarrow \infty$ imposed in Ref. [44] as

$f(r) \sim 2 \sin \left(k r-\frac{\ell \pi}{2}+\delta_{\ell}\right)$,

and comparing Eq. (33), the phase shift and the normalization factor can be found. The result is

$\delta_{\ell}=\frac{\pi}{2}(\ell+1)+\arg \left[\frac{\Gamma\left(\eta_{3}-\eta_{1}-\eta_{2}\right)}{\Gamma\left(\eta_{3}-\eta_{1}\right) \Gamma\left(\eta_{3}-\eta_{2}\right)}\right]$

and

$N=\frac{1}{\left[\Gamma\left(\eta_{3}\right)\right]^{2}}\left|\frac{\Gamma\left(\eta_{3}-\eta_{1}-\eta_{2}\right)}{\Gamma\left(\eta_{3}-\eta_{1}\right) \Gamma\left(\eta_{3}-\eta_{2}\right)}\right|$.

It can be seen that when $\alpha=1$ the results are the same as the Dirac equation in Minkowski spacetime. In this case, the generalized spin-orbit operator in Eq. (13) reduces to

$\kappa= \pm\left(j+\frac{1}{2}\right)$

where $\gamma=\kappa$. Therefore we can obtain the phase shifts and the normalization factor in flat spacetime in this case if we rewrite the Dirac equation in the flat spacetime by standard Dirac matrices. As expected our result is the limit of Eqs. (35) and (36) when $\alpha=1$.

\section{Bound states analysis}

The Hulthén potential also admits bound state solutions. In order to find the bound states energies, let us analyze the 
scattering $S$-matrix. It is well known that poles of the $S$ matrix in the upper half of the complex plane are associated with the bound states. Using Eq. (35), the $S$-matrix can be written as

$$
\begin{aligned}
S_{\ell}= & e^{2 i \delta_{\ell}} \\
= & e^{i \pi(\ell+1)} e^{2 i \arg \left[\Gamma\left(\eta_{3}-\eta_{1}-\eta_{2}\right)\right]} \\
& \times e^{-2 i \arg \left[\Gamma\left(\eta_{3}-\eta_{1}\right)\right]} e^{-2 i \arg \left[\Gamma\left(\eta_{3}-\eta_{2}\right)\right]} .
\end{aligned}
$$

Therefore, the poles of the $S$-matrix are given by the poles of the gamma functions $\Gamma\left(\eta_{3}-\eta_{1}\right)$ and $\Gamma\left(\eta_{3}-\eta_{2}\right)$. In this manner, based on the relations $\eta_{3}-\eta_{2}=\eta_{1}^{*}, \eta_{3}-\eta_{1}=\eta_{2}^{*}$ and $\eta_{3}-\eta_{2}-\eta_{1}=\left(\eta_{1}+\eta_{2}-\eta_{3}\right)^{*}=2 i k / \omega$, we are looking for the poles of

$\Gamma\left(\kappa_{(\alpha)}-\frac{i k}{\omega} \pm \sqrt{\frac{2(E+M) \xi}{\omega}+\frac{k^{2}}{\omega^{2}}}\right)$.

The gamma function $\Gamma(z)$ has poles at $z=-n$, where $n$ is a non-negative integer. Then the bound state energies are given by

$k^{2} \equiv E^{2}-M^{2}=-\frac{\left[\left(n+\kappa_{(\alpha)}^{2} \omega-2(E+M) \xi\right]^{2}\right.}{4\left(n+\kappa_{(\alpha)}^{2}\right)}$,

whit $n=0,1,2, \ldots$.

\section{Conclusion}

In this work we considered the Dirac equation in curved spacetime and the topology of spacetime in order to describe physics of the system in the presence of the gravitational fields of a cosmic string. We obtained the solution of the Dirac equation in the curved spacetime by considering vector and scalar potentials. We considered the scattering states of the Dirac equation under the Hulthén potential and obtained as scattering phase shifts. From the poles of the scattering $S$ matrix we determined the bound state energies. When $\alpha=1$, we recover the general solution of the Dirac equation in usual spherical coordinates, as we should.

Acknowledgements With great pleasure the authors thank the kind referee for helpful comments. FMA acknowledges funding from the Conselho Nacional de Desenvolvimento Científico e Tecnológico (CNPq), Grants No. 460404/2014-8 and No. 311699/2014-6. EOS acknowledges funding from CNPq, Grants No. 482015/2013-6, No. 306068/2013-3, No. 476267/2013-7, FAPEMA Grant No. 01852/14 (PRONEM) and FAPES.

Open Access This article is distributed under the terms of the Creative Commons Attribution 4.0 International License (http://creativecomm ons.org/licenses/by/4.0/), which permits unrestricted use, distribution, and reproduction in any medium, provided you give appropriate credit to the original author(s) and the source, provide a link to the Creative Commons license, and indicate if changes were made.

Funded by SCOAP $^{3}$.

\section{References}

1. I. Lawrie, in A Unified Grand Tour of Theoretical Physics, Third Edition (Taylor \& Francis, London, 2012)

2. N.D. Birrell, P.C.W. Davies, Quantum Fields in Curved Space (Cambridge Univ. Press, Cambridge, 1984)

3. E.R. Bezerra de Mello, J. High Energy Phys. 2004(06), 016 (2004). doi:10.1088/1126-6708/2004/06/016

4. W. Greiner, in Relativistic Quantum Mechanics. Wave Equations (Springer Science Business Media, 2000). doi:10.1007/ 978-3-662-04275-5

5. E.R. Figueiredo Medeiros, E.R. Bezerra de Mello, Eur. Phys. J. C 72(6), 2051 (2012). doi:10.1140/epjc/s10052-012-2051-9

6. J.h. Wang, K. Ma, K. Li, Phys. Rev. A 87(3), 032107 (2013). doi:10. 1103/PhysRevA.87.032107

7. D. Chowdhury, B. Basu, Phys. Rev. D 90, 125014 (2014). doi:10. 1103/PhysRevD.90.125014

8. O. Panella, S. Biondini, A. Arda, J. Phys. A 43(32), 325302 (2010). doi:10.1088/1751-8113/43/32/325302

9. G.d.A. Marques, V.B. Bezerra, S.H. Dong, Mod. Phys. Lett. A 28(31), 1350137 (2013). doi:10.1142/S021773231350137X

10. C.R. Hagen, D.K. Park, Ann. Phys. (NY) 251(1), 45 (1996). doi:10. 1006/aphy.1996.0106

11. V. Khalilov, Eur. Phys. J. C 73(8), 1 (2013). doi:10.1140/epjc/ s10052-013-2548-x

12. O. Aydogdu, R. Sever, Phys. Lett. B 703(3), 379 (2011). doi:10. 1016/j.physletb.2011.08.010

13. Q. Liu, Z.M. Niu, J.Y. Guo, Phys. Rev. A 87, 052122 (2013). doi:10. 1103/PhysRevA.87.052122

14. C.V. Sukumar, J. Phys. A 18(12), L697 (1985)

15. L.B. Castro, A.S. de Castro, Phys. Src. 77(4), 045007 (2008). doi:10.1088/0031-8949/77/04/045007

16. L.B. Castro, A.S. de Castro, J. Phys. A 40(2), 263 (2007). doi:10. 1088/1751-8113/40/2/005

17. L.B. Castro, A.S. de Castro, Phys. Scr. 75(2), 170 (2007). doi:10. 1088/0031-8949/75/2/009

18. A.S. de Castro, M. Hott, Phys. Lett. A 351(6), 379 (2006). doi:10. 1016/j.physleta.2005.11.033

19. H. Egrifes, R. Sever, Phys. Lett. A 344, 117 (2005). doi:10.1016/ j.physleta.2005.06.061

20. H. Kleinert, I. Mustapic, J. Math. Phys. 33(2), 643 (1992). doi:10. $1063 / 1.529800$

21. H. Weyl, Phys. Rev. 77(5), 699 (1950). doi:10.1103/physrev.77. 699

22. L. Parker, Phys. Rev. Lett. 44(23), 1559 (1980). doi:10.1103/ physrevlett.44.1559

23. L. Parker, Phys. Rev. D 22(8), 1922 (1980). doi:10.1103/physrevd. 22.1922

24. M. Hosseinpour, H. Hassanabadi, Int. J. Mod. Phys. A 30(21), 1550124 (2015). doi:10.1142/s0217751x15501249

25. Ph. de Sousa Gerbert, Phys. Rev. D 40(4), 1346 (1989). doi:10. 1103/PhysRevD.40.1346

26. I.I. Cotaescu, M. Visinescu, in Progress in General Relativity and Quantum Cosmology Research, Nova Science, N.Y. (2007 (2004), pp. 109-166

27. C.A. de Lima Ribeiro, C. Furtado, F. Moraes, Europhys. Lett. 62(3), 306 (2003). doi:10.1209/epl/i2003-00396-3

28. C. Furtado, F. Moraes, Phys. Lett. A 188(4-6), 394 (1994). doi:10. 1016/0375-9601(94)90482-0 
29. C. Furtado, B.G. da Cunha, F. Moraes, E. de Mello, V. Bezzerra, Phys. Lett. A 195(1), 90 (1994). doi:10.1016/ 0375-9601(94)90432-4

30. C. Furtado, F. Moraes, Europhys. Lett. 45(3), 279 (1999). doi:10. 1209/ep1/i1999-00159-8

31. G. de A Marques, C. Furtado, V.B. Bezerra, F. Moraes, J. Phys. A 34(30), 5945 (2001). doi:10.1088/0305-4470/34/30/306

32. E.R.F. Medeiros, E.R.B. de Mello, Eur. Phys. J. C 72(6), 2051 (2012). doi:10.1140/epjc/s10052-012-2051-9

33. E.B. de Mello, V. Bezerra, A. Saharian, H. Harutyunyan, Phys. Rev. D 91(6), 064034 (2015). doi:10.1103/physrevd.91.064034

34. M.M. de Sousa, R. Ribeiro, E.B. de Mello, Phys. Rev. D 93(4), 043545 (2016). doi:10.1103/physrevd.93.043545

35. M.M. de Sousa, R. Ribeiro, E.B. de Mello, Phys. Rev. D 95(4), 045005 (2017). doi:10.1103/physrevd.95.045005

36. H.F. Mota, K. Bakke, Phys. Rev. D 89(2), 027702 (2014). doi:10. 1103/physrevd.89.027702

37. C. Filgueiras, F. Moraes, Phys. Lett. A 361(1-2), 13 (2007). doi:10. 1016/j.physleta.2006.09.030

38. M. Alford, J. March-Russell, F. Wilczek, Nucl. Phys. B 328(1), 140 (1989). doi:10.1016/0550-3213(89)90096-5

39. F.M. Andrade, E.O. Silva, M. Pereira, Phys. Rev. D 85(4), 041701(R) (2012). doi:10.1103/PhysRevD.85.041701

40. F.M. Andrade, E.O. Silva, M. Pereira, Ann. Phys. (NY) 339, 510 (2013). doi:10.1016/j.aop.2013.10.001

41. F.M. Andrade, E.O. Silva, Eur. Phys. J. C 74, 3187 (2014). doi:10. 1140/epjc/s10052-014-3187-6

42. A. Vilenkin, E.P.S. Shellard, Cosmic Strings and Other Topological Defects (Cambridge University Pres, Canbridge, 2000)

43. N.G. Marchuk, Nuovo Cimento 115B, 11 (2000)

44. L.D. Landau, E.M. Lifschitz, Quantum Mechanics (Pergamon, Oxford, 1981)

45. G.A. Marques, V.B. Bezerra, Phys. Rev. D 66(10), 105011 (2002). doi:10.1103/PhysRevD.66.105011
46. R. Bausch, R. Schmitz, L.A. Turski, Phys. Rev. Lett. 80, 2257 (1998). doi:10.1103/PhysRevLett.80.2257

47. E. Aurell, J. Phys. A 32(4), 571 (1999). doi:10.1088/0305-4470/ 32/4/004

48. K. Kawamura, Z. Physik B 29(2), 101 (1978). doi:10.1007/ bf01313193

49. C. Furtado, V. Bezerra, F. Moraes, Phys. Lett. A 289(3), 160 (2001). doi:10.1016/S0375-9601(01)00615-6

50. C. Furtado, V.B. Bezerra, F. Moraes, Europhys. Lett. 52(1), 1 (2000). doi:10.1209/epl/i2000-00396-3

51. P.S. Letelier, Class. Quantum Grav. 12(2), 471 (1995). doi:10.1088/ 0264-9381/12/2/016

52. D.V. Gal'tsov, P.S. Letelier, Phys. Rev. D 47(10), 4273 (1993). doi: $10.1103 /$ physrevd. 47.4273

53. A. Vilenkin, Phys. Rep. 121(5), 263 (1985). doi:10.1016/ 0370-1573(85)90033-x

54. B. Linet, Gen. Relat. Gravit. 17(11), 1109 (1985). doi:10.1007/ bf00774211

55. S.H. Dong, W.C. Qiang, G.H. Sun, V.B. Bezerra, J. Phys. A 40(34), 10535 (2007). doi:10.1088/1751-8113/40/34/010

56. P. de Sousa Gerbert, R. Jackiw, Commun. Math. Phys. 124, 229 (1989). doi:10.1007/BF01219196

57. W.B. Perkins, A.C. Davis, Nucl. Phys. B 349(1), 207 (1991). doi:10.1016/0550-3213(91)90194-3

58. P. Górnicki, Ann. Phys. (NY) 202(2), 271 (1990). doi:10.1016/ 0003-4916(90)90226-e

59. W. Perkins, L. Perivolaropoulos, A.C. Davis, R. Brandenberger, A. Matheson, Nucl. Phys. B 353(1), 237 (1991). doi:10.1016/ 0550-3213(91)90509-v

60. W.C. Qiang, S.H. Dong, Phys. Lett. A 368(1-2), 13 (2007). doi:10. 1016/j.physleta.2007.03.057

61. I.S. Gradshteyn, I.M. Ryzhikif, Table of Integrals, Series, and Products, seventh edition. (Academic, 2007) 